\title{
Design and Characterization of Single-Layer Step-Bridge Structure for Out-of-Plane Thermal Actuator
}

\author{
Wen-Chih Chen, Po-I Yeh, Chih-Fan Hu, and Weileun Fang, Member, IEEE, Member, ASME
}

\begin{abstract}
This paper presents the design and fabrication of a single-layer out-of-plane thermal actuator. The step-bridge structure design enables bending and then buckling of the actuator in the out-of-plane direction by Joule heating. Moreover, the moving direction of the actuator can be specified by the step structure. In summary, the step-bridge actuator design has the following five merits: 1) The load-deflection relation is easily tuned; 2) the bistable buckling behavior is prevented; 3 ) the unwanted vibration modes can be suppressed; 4) the delamination problem is prevented; and 5) the bridge structure is stiffer and more stable. The actuator and its application on a lens positioning stage have been implemented using $p^{++}$Si layer by bulk micromachining. It demonstrates that a typical actuator would move upward with an amplitude near $13 \mu \mathrm{m}$ when driven at $54 \mathrm{~mW}$.

[2007-0084]
\end{abstract}

Index Terms-Buckling, microelectromechanical systems (MEMS), step-bridge, thermal actuator.

\section{INTRODUCTION}

A MICROACTUATOR is a key component in enabling motion of microsystems. A microactuator is also frequently employed to precisely control the position of microstructures. Currently, various available approaches, such as electrostatic, piezoelectric, magnetic, and electrothermal techniques, have been exploited to drive the microactuators. The design considerations of microactuators are focused on the output force and displacement, driving voltage, power consumption, reliability, etc. In addition, the fabrication process and the capability of integration with other microcomponents are also critical considerations for microactuators. The electrothermal actuators have the following advantages: low driving voltage, large output force, and large output displacement [1]-[4]. The existing reports also show the following advantages: simple fabrication process and easy integration with other microelectromechanical systems (MEMS) components for some electrothermal actuators [5], [6]. In applications, the electrothermal actuators have been applied to drive various MEMS devices [7]-[9].

Manuscript received April 16, 2007; revised July 30, 2007. This work was supported in part by the National Science Council, Taiwan, R.O.C., under Grant NSC 95-2221-E-007-158. Subject Editor D. Cho.

W.-C. Chen, P.-I Yeh, and C.-F. Hu are with the Department of Power Mechanical Engineering, National Tsing Hua University, Hsinchu 30013, Taiwan, R.O.C.

W. Fang is with the Department of Power Mechanical Engineering and also with the Institute of Nanoengineering and Microsystems, National Tsing Hua University, Hsinchu 30013, Taiwan, R.O.C. (e-mail: fang@ @me.nthu.edu.tw).

Color versions of one or more of the figures in this paper are available online at http://ieeexplore.iee.org.

Digital Object Identifier 10.1109/JMEMS.2007.906761
There are many available in-plane electrothermal actuators, such as U-shaped [2] and V-shaped actuators [3]. The former is frequently employed in the multi-user MEMS processes and is readily integrated with various existing surface micromachined components for many applications, as discussed in [8], [10], and [11]. The latter can easily be fabricated and integrated with other components on silicon on insulator (SOI) wafer, and it accordingly has various applications, as discussed in [9] and [12]. Nevertheless, reliability becomes a critical issue for an electrothermal actuator made of nonsingle-crystal-silicon materials [13]. On the other hand, the existing out-of-plane thermal actuator is usually formed by a multilayer structure. For instance, the bilayer electrothermal actuator, which comprises two materials of different coefficients of thermal expansion, employs the bimorph effect to generate out-of-plane motion [1]. However, the multilayer actuator experiences a shear force at the interface of different layers during actuation. The delamination of thin films will take place after a long-term operation, and it consequently decreases the lifetime of the actuator.

To overcome the drawback that is inherent in a multilayer actuator, a cantilever-type out-of-plane electrothermal actuator of single-layer structure has been reported in [14]. However, the small stiffness of the cantilever structure limits the application of the actuator. In this paper, a novel single-layer step-bridge actuator is designed and implemented. The bridge structure has both of its two ends clamped to the substrate. Compared with a cantilever which has only one end fixed to the substrate, the bridge structure is stiffer [15] and more stable during operation. According to the design of the step structure, the loaddeflection relation from prebuckling to postbuckling is easily tuned, and the bistable behavior of buckling is also prevented. Moreover, the unwanted motion can be suppressed by stepbridge design so as to provide pure linear or torsional output displacements.

\section{Design And Analysis}

This paper presents a novel step-bridge structure that is shown in Fig. 1 to act as the single-layer out-of-plane electrothermal actuator. The step-bridge is a clamped-clamped beam with two step structures that are symmetrically distributed about the beam center. The current-induced Joule heating will lead to the thermal expansion of the structure and will cause bending, as well as buckling deformations, of the step-bridge. The maximum out-of-plane deformation at the center of the step-bridge provides the output displacement of the actuator. 
(a)

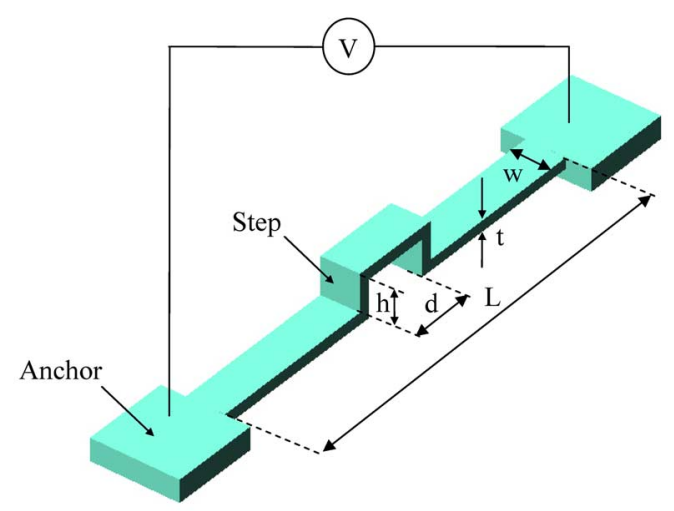

(b)

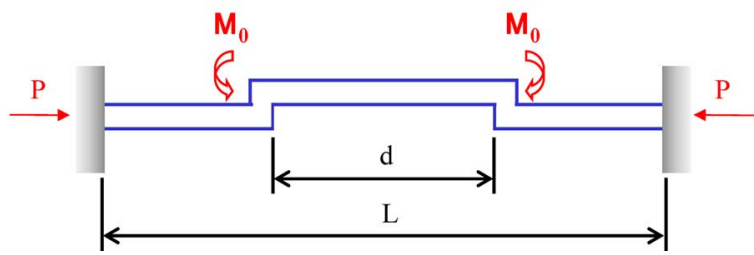

Fig. 1. (a) Schematic design of the step-bridge actuator. (b) Mechanics model of the actuator.

\section{A. Concept and Design}

The step-bridge structure in Fig. 1 will experience a thermal expansion that is caused by Joule heating when applying with current. As shown in the step design, the structure will simultaneously be subjected to an axial load $P$ and a bending moment $M_{0}$ by its thermal expansion, resulting in the deformation of the step-bridge. The step-bridge is initially bent by the bending moment during heating, and the bending deformation is gradually increased with the thermal load. As the thermal load reaches a critical value, the bent step-bridge will further be buckled by the axial load. In summary, the static out-of-plane displacement of the present thermal actuator is caused by both buckling and bending deformations of the step-bridge structure during $\mathrm{dc}$ actuation. The output displacement of the out-of-plane actuator can be tuned by varying not only the length $L$ and thickness $t$ of the bridge but also the height $h$ and length $d$ of the step. In short, the step structure acts as the imperfection of the bridge during buckling [16] that prevents the bistable behavior, and it enables the smooth deformation of the bridge from prebuckling to postbuckling by Joule heating.

In a similar manner, the dynamic out-of-plane displacement of the thermal actuator is also available after driving the stepbridge structure with ac current. The shape of the step-bridge (i.e., the location of the step) that is designed in this paper is symmetric about the midpoint of the beam, as shown in Fig. 1 . The bending moment $M_{0} \sin \omega t$ that is induced by a harmonic ac current of frequency $\omega / 2$ is applied at the steps, and it has the same distance $d / 2$ away from the midpoint of the step-bridge. Moreover, these two bending moments have the same magnitude, but have opposite directions. Thus, the bending moments that act on the steps can also be modeled as an equivalent harmonic load $P \sin \omega t$ that is applied at the midpoint of the step-bridge. In other words, the harmonic load is (a)

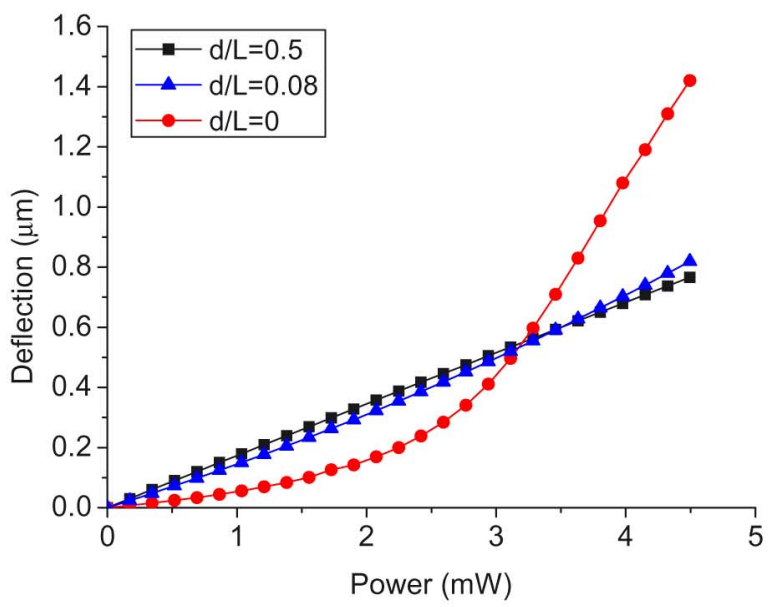

(b)

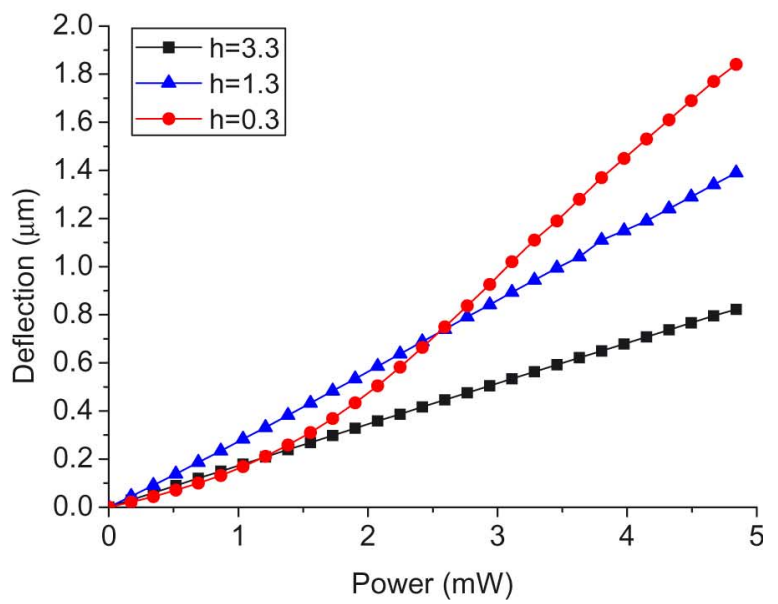

Fig. 2. Load-deflection simulation results of the step-bridge actuator versus the input power (a) for three different $d / L$ designs, with $h=3.3$, and (b) for three different $h$ designs, with $d / L=0.5$.

applied at the node in even vibration modes of the step-bridge. This characteristic has been exploited in the present paper to suppress the unwanted dynamic responses while driving the actuator.

\section{B. Finite-Element Simulation}

The static and dynamic characteristics of the step-bridge actuator have been investigated by means of the finite-element simulation. Fig. 1(b) shows the model of the step-bridge actuator. The performance of the thermal actuator can be tuned by varying the dimensions of the beam length $L$, step structure length $d$, and step height $h$. In this paper, the length of the stepbridge $L$ was specified, and the influence of the step structures with different $h$ and $d / L$ was examined by using a commercial finite-element software. In order to compare it with the experimental results, the thin-film material and substrate that is employed in this model was a single crystal silicon. The material properties that are used in the finite-element method (FEM) model were as follows: Young's modulus $E=125 \mathrm{GPa}$, density $D=2.33 \times 10^{3} \mathrm{~kg} / \mathrm{m}^{3}$, coefficient of thermal expansion $\alpha=2.5 \times 10^{-6}$, thermal conductivity $\mathrm{Kp}=157 \mathrm{~W} /\left(\mathrm{m}{ }^{\circ} \mathrm{C}\right)$, 
TABLE I

Predicted Mode Shapes During Free Vibration and the Vibration Modes That Are Excited by Harmonic Thermal Loads

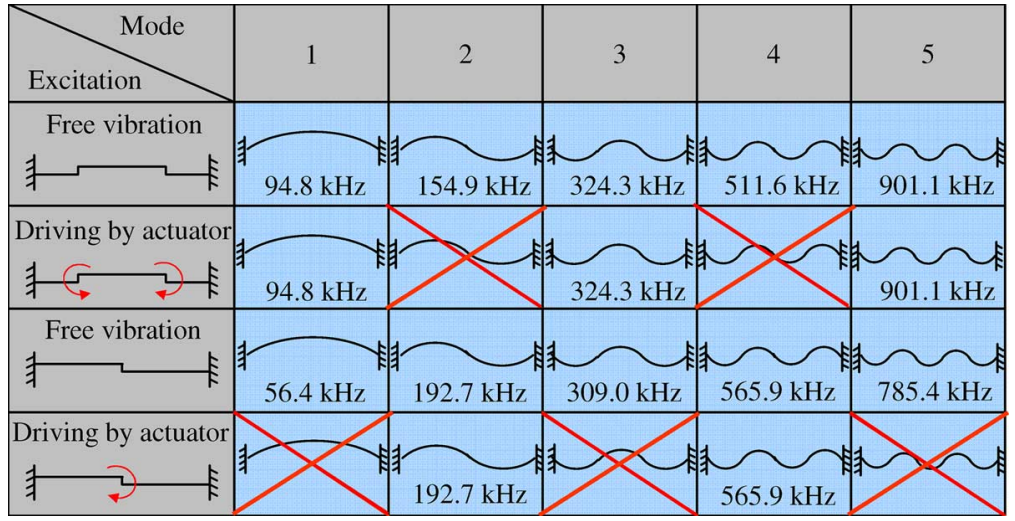

and resistivity $\rho=1.1 \times 10^{-5} \Omega \cdot \mathrm{m}$. The actuator had the dimensions of length $L=600 \mu \mathrm{m}$, thickness $t=2.2 \mu \mathrm{m}$, and width $w=27 \mu \mathrm{m}$. In addition, the step structures of different $d / L$ and step heights $h$ were discussed.

The simulation results in Fig. 2(a) depict the deflection amplitude of the step-bridge actuator versus the input power for three different $d / L$ designs (i.e., $d / L=0,0.08$, and 0.5 ). It indicates that the operating characteristic of the actuator, such as the maximum deflection and the load-deflection relation, can be tuned by varying the length of the step structure $d$ while the total beam length $L$ remains unchanged. When the length $d$ is approaching zero or length $L$, the load-deflection characteristic of the present thermal actuator is similar to that of an ideal bridge structure, and the influence of the buckling effect becomes more significant. The deflection at the low input power region (prebuckling region) shows the bending but not buckling deformation of the step-bridge, which is contributed by the step design. Fig. 2(b) shows the deflection amplitude of the stepbridge actuator versus the input power for three different step heights $h$ (i.e., $h=0.3,1.3$, and $3.3 \mu \mathrm{m}$ ). It demonstrates that the operating characteristic of the actuator can also be tuned by varying the step height $h$. The buckling effect becomes more significant when the step height gets smaller. In short, the simulation reveals that the output displacement of the actuator is contributed by both bending and buckling deformations of the step-bridge structure.

The dynamic characteristics of the step-bridge actuators are also investigated using the FEM. Table I shows the predicted mode shapes during free vibration and the vibration modes that are excited by harmonic thermal loads. The first five natural modes of the actuator are associated with resonant frequencies of $94.8,154.9,324.3,511.6$, and $901.1 \mathrm{kHz}$, respectively. However, the harmonic thermal loads can only excite the vibration modes of $94.8,324.3$, and $901.1 \mathrm{kHz}$. It shows that the even natural modes of the step-bridge structure are suppressed during thermal actuation. The simulation results demonstrate the feasibility of the design concept. According to the modesuppressing characteristic, the unwanted torsional motion that is induced by the second mode of the actuator can be prevented. Therefore, it can be used for some applications which require pure out-of-plane linear motion.

\section{Theoretical Analysis}

The out-of-plane deflection of the step-bridge is contributed by the pure bending and buckling of the structure. The purebending and buckling deformations are caused by the bending moment $M_{0}$ and the axial load $P$ that are shown in Fig. 1(b), respectively. The prebuckling and postbuckling deformations of the step-bridge structure during actuation are also investigated through the theoretical analysis. Since the step is a movable and deformable structure, the deformation of the step-bridge actuator will be influenced by the step height $h$ and the input power. In this paper, the analysis will only focus on the variation of the actuator output with the parameter $d / L$ at a particular $h$ and input power. Thus, the actuator deformations due to $d / L$, bending, and buckling effects can qualitatively be discussed.

1) Prebuckling Deformation: In the prebuckling state, the out-of-plane deformation of the step-bridge structure is mainly introduced by the bending moment $M_{0}$ that is shown in Fig. 1(b). Thus, the prebuckling deformation amplitude $U_{\text {pre }}$ of the actuator at the midpoint of the structure [i.e., at $L / 2$ in Fig. 1(b)] can be expressed as

$$
U_{\text {pre }}=\frac{M_{0} L^{2}}{8 E I}\left(\frac{d}{L}-\left(\frac{d}{L}\right)^{2}\right) H_{1}
$$

where $E$ and $I$ are the Young's modulus and the moment of inertia, respectively. To simplify the analysis, the parameter $H_{1}$ is introduced into (1) to represent the influence of the step on $U_{\text {pre }}$, and $H_{1}$ is a function of $h$ and the input power. Thus, the variation of the prebuckling deflection amplitude $U_{\text {pre }}$ with respect to the ratio of $d / L$ is determined by (1). The maximum deflection of the actuator will occur at $d / L=0.5$ after differentiating (1) with respect to $d$.

2) Postbuckling Deformation: In the postbuckling state, the out-of-plane deformation of the step-bridge structure is mainly determined by the eccentric axial load $P$ that is shown in Fig. 3(a). The eccentric distance of the load $P$ is determined by the step height $h$. As shown in Fig. 3(b), the buckling deformation $U_{\text {post }}$ of the step-bridge with length $L$ is superposed by the buckling deformations of the bridges with lengths of $d$ and $L-d$. These two bridges are under the same eccentric axial 
(a)

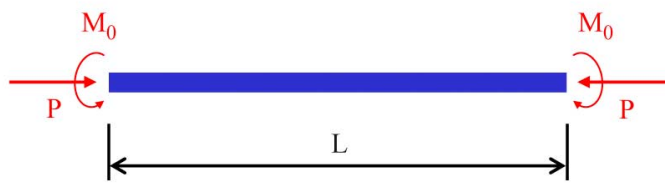

(b)
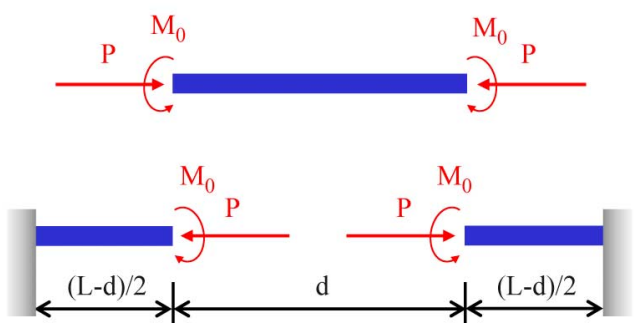

Fig. 3. Mechanics model of the step-bridge structure with the eccentric axial load $P$ for (a) $d / L=0$ and (b) $0<d / L<1$.

load of $P$. Therefore, from the buckle beam theory of eccentric axial load, the deflection of the step-bridge becomes [15]

$U_{\text {post }}=\left(\frac{1}{\cos \left(\frac{L}{2} \sqrt{\frac{P}{E I}} \frac{d}{L}\right)}+\frac{1}{\cos \left(\frac{L}{2} \sqrt{\frac{P}{E I}}\left(1-\frac{d}{L}\right)\right)}-2\right) H_{2}$

where the parameter $\mathrm{H}_{2}$ represents the influence of the step on $U_{\text {post }}$, and $\mathrm{H}_{2}$ is also a function of $h$ and the input power. As the length ratio $d / L$ approaches to zero or one, the shape of the step-bridge is similar to the ideal clamped-clamped beam, and its buckling deformation becomes

$$
U_{\text {post }}=\left(\frac{1}{\cos \left(\sqrt{\frac{P}{E I}} \frac{L}{2}\right)}-1\right) H_{2} .
$$

In addition, (2) has a minimum $U_{\text {post }}$ at $d / L=0.5$, and the buckling deflection of the step-bridge is yielded as

$$
U_{\text {post }}=2\left(\frac{1}{\cos \left(\sqrt{\frac{P}{E I}} \frac{L}{4}\right)}-1\right) H_{2} .
$$

Fig. 4 summarizes the characteristics of the step-bridge deformation with respect to the length ratio $d / L$. The concave downward curve is derived from (1) at a particular step height $h$ and input power. It is concluded that the maximum output of the actuator will occur at $d / L=0.5$ when the pure-bending behavior dominates the step-bridge deflection, as indicated by the prebuckling deflection curve. On the other hand, the concave upward curve is derived from (2) at a particular $h$ and input power. However, the latter case experiences a larger input power to allow buckling to occur. The actuator has a minimum output at $d / L=0.5$ when the buckling effect dominates the beam deflection, as indicated by the postbuckling deflection curve.

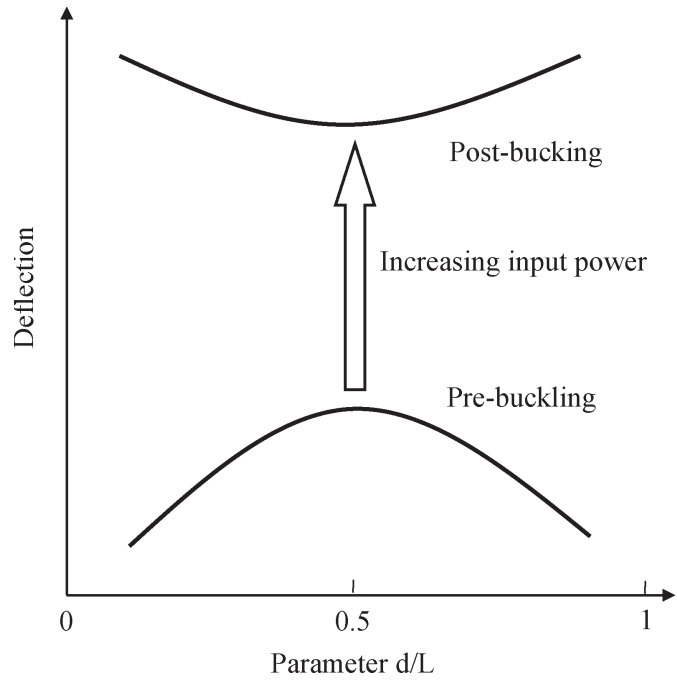

Fig. 4. Characteristics of the step-bridge deformation with respect to the length ratio $d / L$

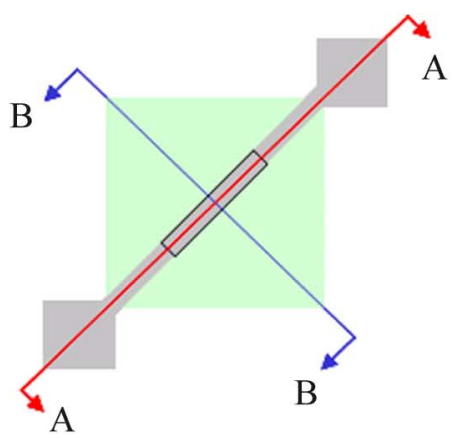

(a)

A-A

B-B

(b)
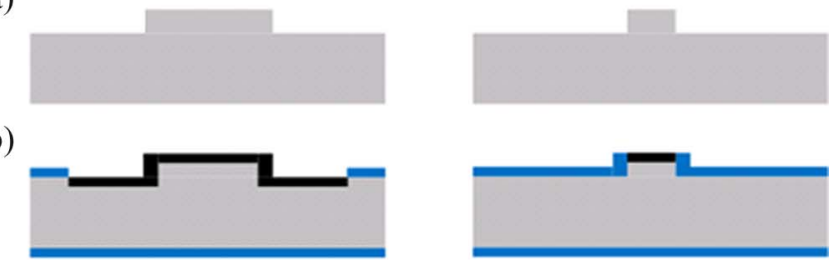

(c)
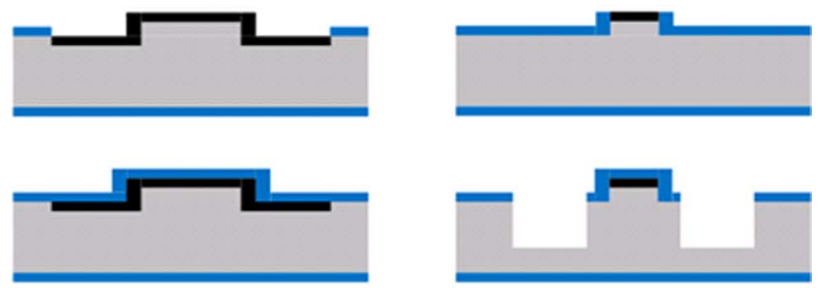

(d)

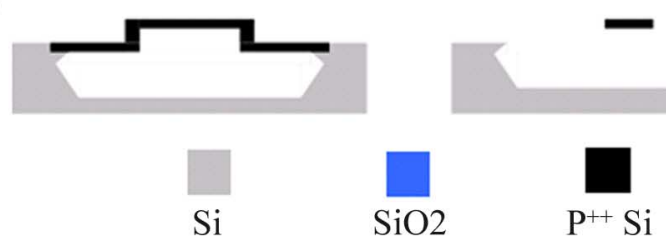

Fig. 5. Fabrication processes of the proposed thermal actuator.

\section{EXPERIMENTS AND RESULTS}

The fabrication processes that contain three masks are shown in Fig. 5. The process began with the first photolithography to define the location and length $d$ of the step structure. As shown in Fig. 5(a), the first reactive ion etching (RIE) was then used 
(a)

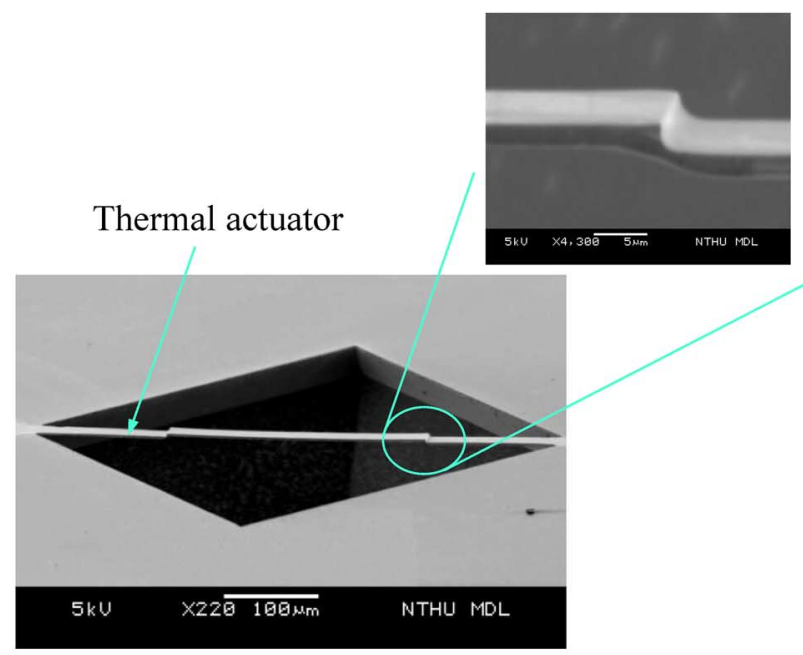

(b)

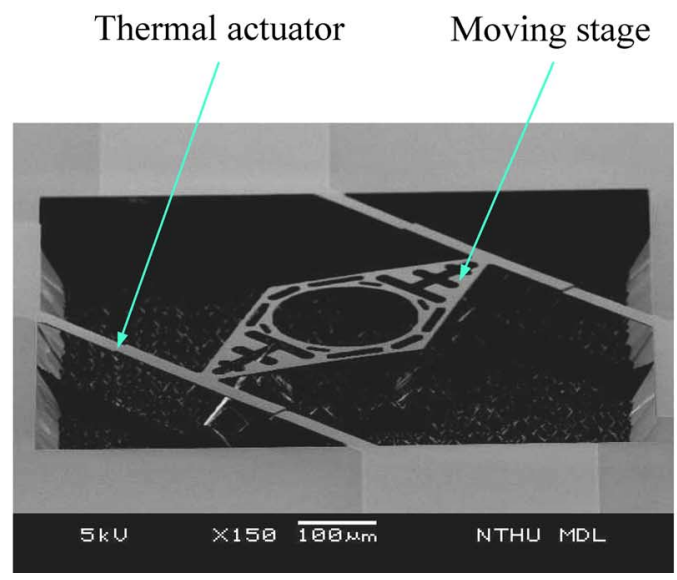

Fig. 6. SEM photos of (a) a step-bridge actuator, where the inset shows the zoom-in photo of the step structure, and (b) a moving stage that is integrated with two step-bridge actuators.

to etch the silicon substrate to define the depth $h$ of the step. Thermal oxide was grown and pattered to define the in-plane shape of the step-bridge actuator. After that, a heavy boron was doped into the substrate without covering by thermal oxide to form the actuator, as shown in Fig. 5(b). The thickness of the single-layer actuator was determined by the $\mathrm{p}^{++}$silicon. After removing the first thermal oxide layer, a second thermal oxide was grown to reduce the residual stress of the $\mathrm{p}^{++}$silicon. This thermal oxide was then patterned to act as the etching mask for the substrate during the following deep RIE process, as shown in Fig. 5(c). This process was used to reduce the etching time of the final bulk Si undercut. Silicon substrate bulk etching was subsequently conducted to release the step-bridge actuator, as shown in Fig. 5(d). Consequently, the actuator was formed by the single-layer boron-doped single crystal silicon. Fig. 6(a) shows a typical fabricated single-layer step-bridge actuator with beam length $L=600 \mu \mathrm{m}$, step structure length $d=300 \mu \mathrm{m}$ (i.e., $d / L=0.5$ ), thickness $t=2.2 \mu \mathrm{m}$, width $w=27 \mu \mathrm{m}$, and step height $h=3.3 \mu \mathrm{m}$. The inset shows the zoom-in photo of the step structure. In addition, Fig. 6(b) shows the integration of the step-bridge actuators with a supporting frame to form a

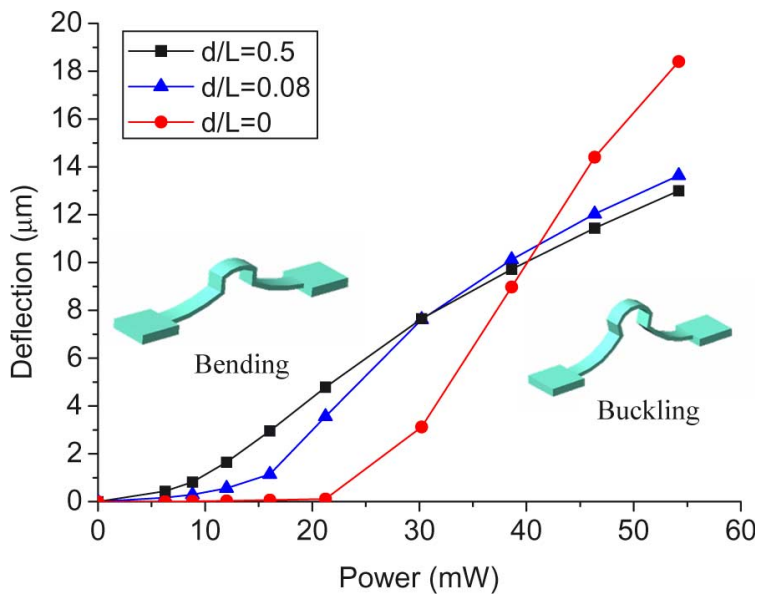

Fig. 7. Typical deflection amplitude versus the input power measured from three actuators of different $d / L(d / L=0,0.08$, and 0.5$)$.

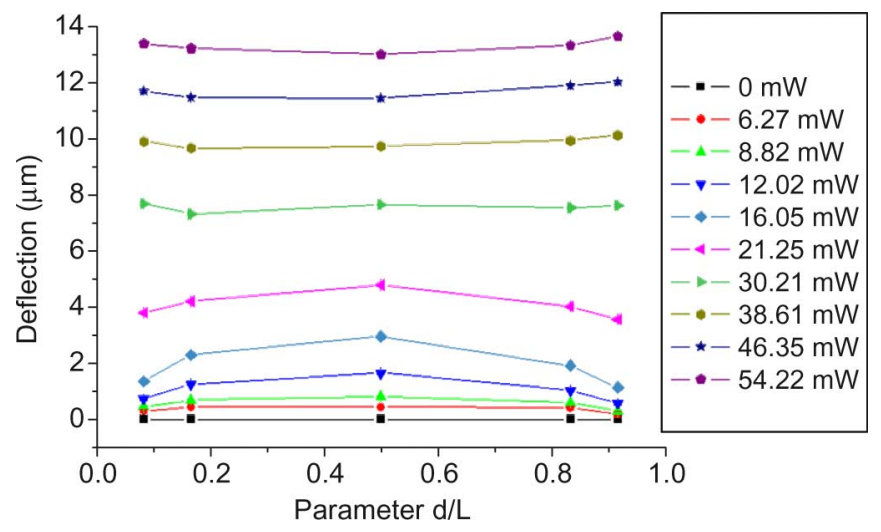

Fig. 8. Effect of the ratio $d / L$ on the deflection amplitude of the step-bridge actuator at different input powers.

micropositioning stage. The position of the supporting frame is controlled by the step-bridge actuators.

The static load-deflection and the dynamic frequency response of the fabricated actuator were measured to characterize its performances. For a static load-deflection test, the actuator was driven by a dc signal from the power supply, and the static out-of-plane deformation of the step-bridge was measured by an optical interferometer. During the tests, various step lengths $d$ were employed to evaluate the performances of the actuator at different $d / L$; however, the rest of the step-bridge dimensions remained the same (i.e., beam length $L=600 \mu \mathrm{m}$, thickness $t=2.2 \mu \mathrm{m}$, width $w=27 \mu \mathrm{m}$, and step height $h=3.3 \mu \mathrm{m}$ ). Fig. 7 shows the typical deflection amplitude versus the input power measured from three actuators of different $d / L$ (i.e., $d / L=0,0.08$, and 0.5$)$. It shows that the actuators with step structure (i.e., $d / L=0.08$ and 0.5 ) moved upward with an amplitude near $13 \mu \mathrm{m}$ when driven at $54 \mathrm{~mW}$, and the actuator with no step structure had an amplitude of $18.4 \mu \mathrm{m}$ at the same input power. Thus, the step structure design successfully enables the tuning of the load-deflection relation of the bridge actuator during the transition from prebuckling to postbuckling. The results qualitatively agree with those predicted from finiteelement simulation in Fig. 2(a).

Fig. 8 shows the effect of the ratio $d / L$ on the deflection amplitude of the step-bridge actuator at different input powers. 


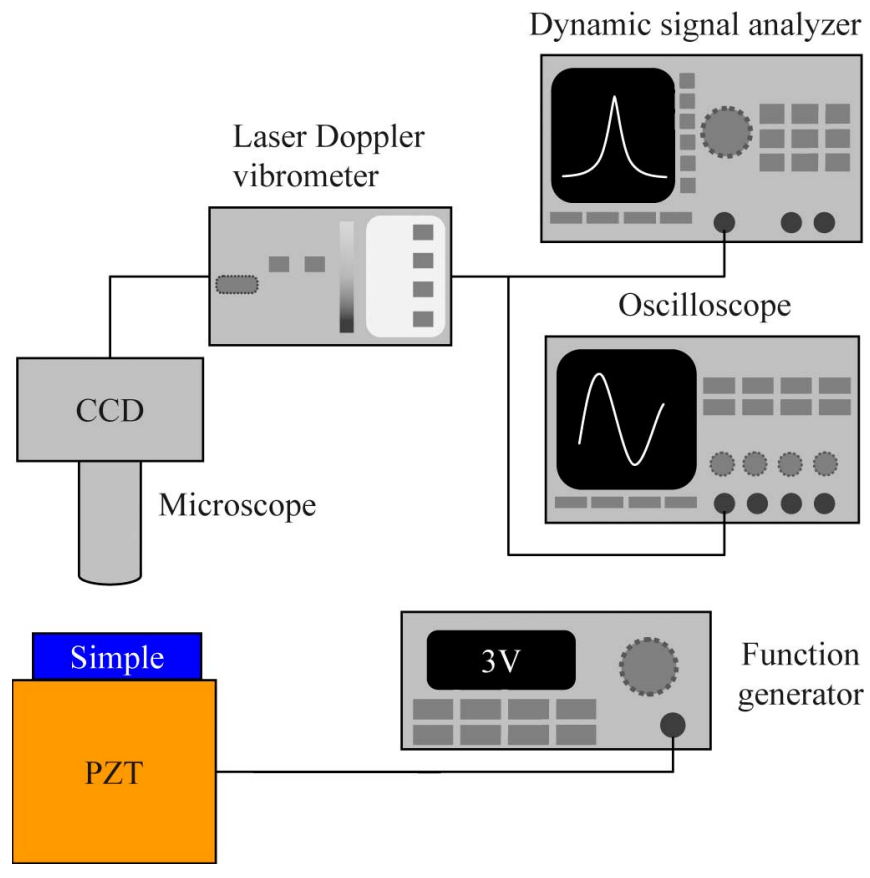

Fig. 9. Experimental setup for dynamic test.

It shows that the actuator with $d / L=0.5$ has a maximum deflection amplitude, while input power is less than about $21.5 \mathrm{~mW}$. However, when the input power is larger than $38.6 \mathrm{~mW}$, the actuator with a ratio of $d / L=0.5$ has a minimum deflection. These characteristics agree with the analysis in Fig. 4. The deflection of the actuator is dominated by the bending effect at low input power and by the buckling effect at high input power. Fig. 8 also shows that the structures with ratios of $d / L$ and $(1-d / L)$ have the same deflection amplitude. This characteristic also agrees with the analysis predicted in (1) and (2).

The experimental setup in Fig. 9 was established for dynamic test. The step-bridge structure was driven by a PZT-shaker to characterize its natural frequencies and mode shapes. In addition, the step-bridge was driven by ac signals to characterize the dynamic frequency response of the present electrothermal actuator. The dynamic response of the actuator was measured by the laser Doppler vibrometer (LDV). Fig. 10(a) shows the typical measured frequency response of the step-bridge structure excited by the PZT-shaker. The first five peaks of the structure are $88.1,186.8,351.7,482.7$, and $730.5 \mathrm{kHz}$, respectively. The mode shapes that are associated with these five natural frequencies are also measured by LDV, as shown in Fig. 10(a). Fig. 10(b) shows the frequency response of the stepbridge actuator after it is driven by an input ac signal. Compared with the structural modes that are excited by the PZT-shaker, the even modes of the step-bridge actuator driven by ac signals were suppressed. The measurement results agree well with the simulation results that are shown in Table I. In conclusion, the present actuators can drop unwanted dynamic responses using the step structure design.

This paper has also demonstrated the integration of the stepbridge structure with other micromachined components to show the potential applications of the actuator. For instance, the SEM photo in Fig. 6 shows the integration of a moving stage with two (a)

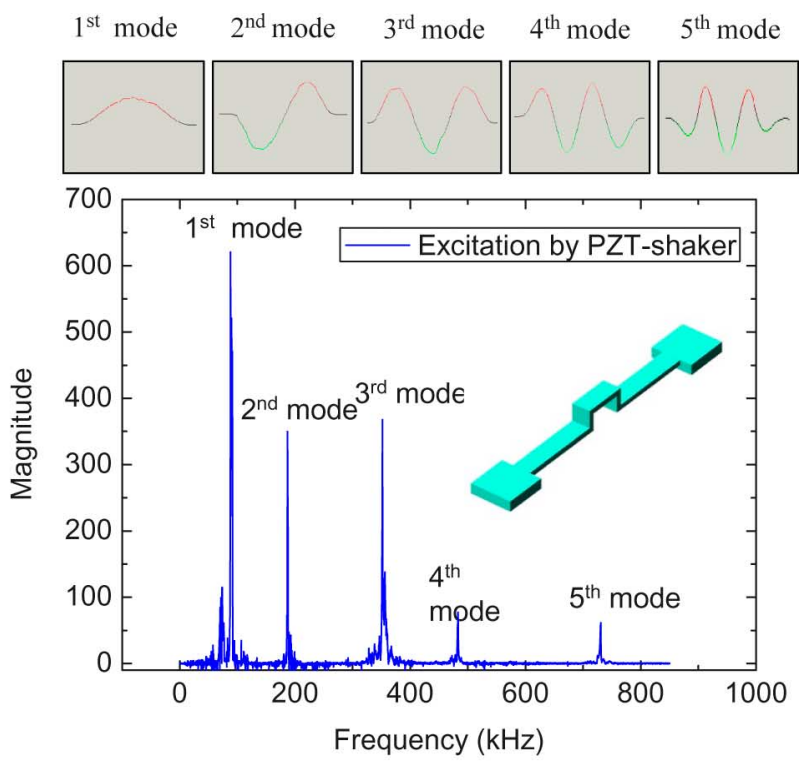

(b)

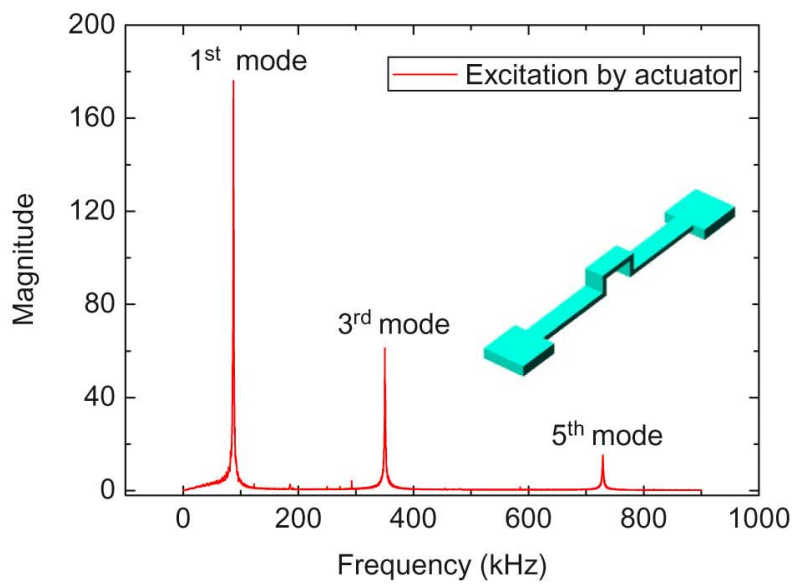

Fig. 10. Typical measured frequency response and mode shapes of the stepbridge actuator excited (a) by an external PZT-shaker and (b) after the actuator is driven by an input ac signal.

step-bridge actuators. Fig. 11(a) further shows the SEM photo of this component after dripping the UV-curable polymer onto the moving stage to form a polymer lens. In this application, the step-bridge actuators were employed to drive the position stage with polymer lens. Fig. 11(b) shows the measured displacement of the position stage at different input powers. The actuator moved upward with a displacement near $13.0 \mu \mathrm{m}$ when driven at $121 \mathrm{~mW}$. Fig. 12 shows the measured frequency response of the polymer-lens position stage. According to the modesuppressing characteristic, the unwanted torsional motion of the lens focusing stage, which is induced by the second mode of the step-bridge actuator, was prevented.

\section{Discussions AND CONCLUSION}

In this paper, a novel single-layer step-bridge out-of-plane thermal actuator is implemented and characterized. A typical load-deflection test demonstrates that the $600-\mu \mathrm{m}$-long actuator moved in the designed direction with an amplitude near $13 \mu \mathrm{m}$ when driven at $54 \mathrm{~mW}$. The output displacement of the actuator 
(a)

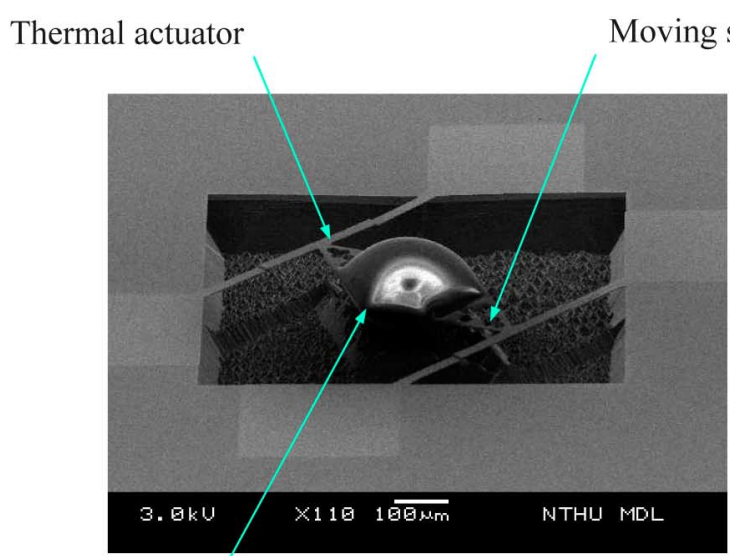

Polymer lens

(b)

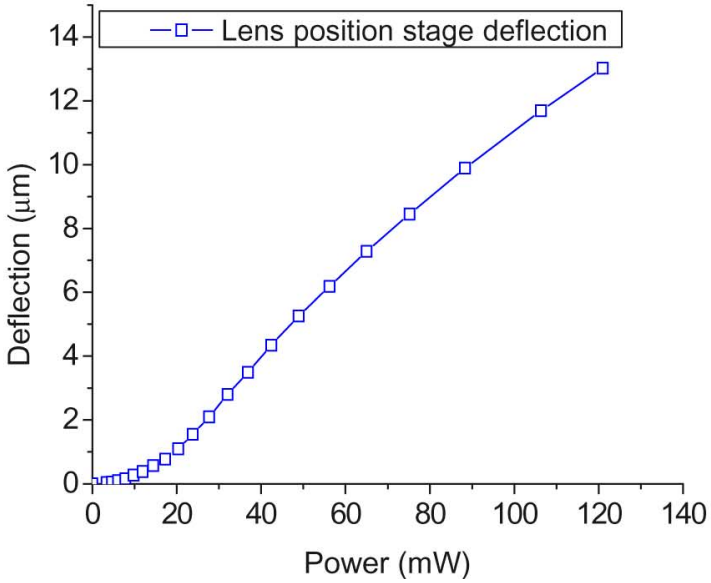

Fig. 11. (a) SEM photo of the moving stage that is integrated with polymer lens. (b) Measured displacement of the polymer-lens position stage at different input powers.

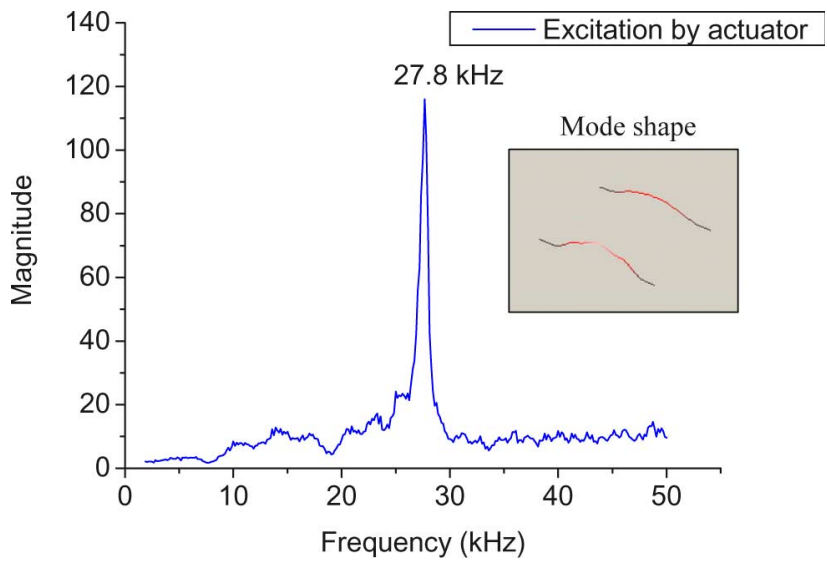

Fig. 12. Measured frequency response of the polymer-lens position stage.

is caused by both bending and buckling deformations of the step-bridge structure, and the moving direction of the actuator output is prescribed by the step structure. In addition, the step height $h$ and step length $d$ have been successfully exploited to tune the linearity and the amplitude of the output displacement of the actuator. Furthermore, this paper also demonstrated the (a)

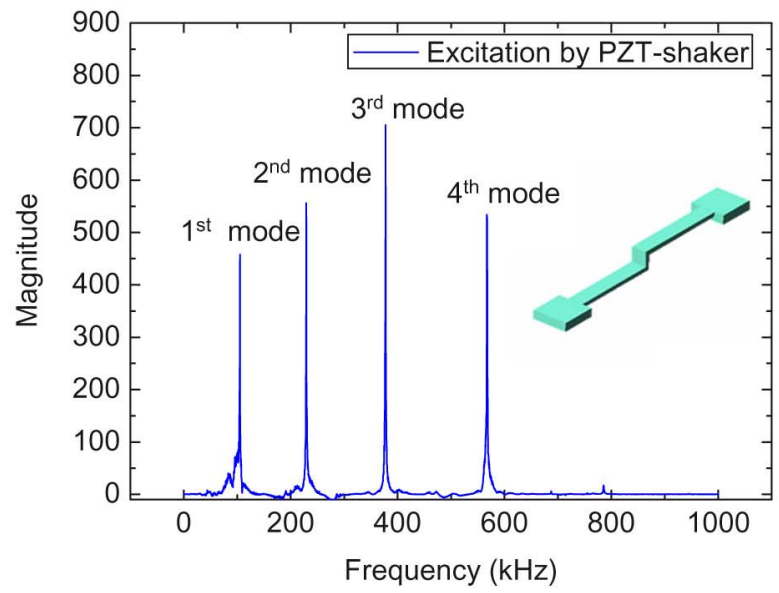

(b)

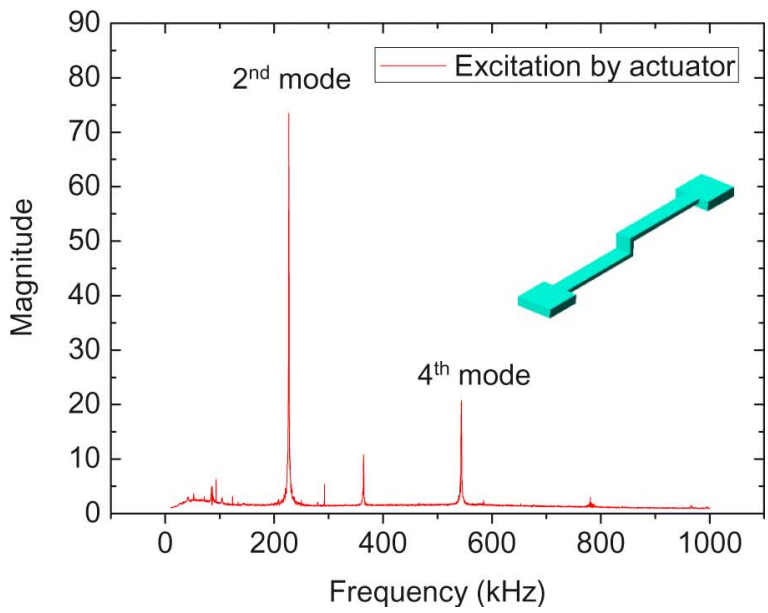

Fig. 13. Typical measured frequency response of the single-step structure (a) by an external PZT-shaker and (b) after the actuator is driven by an input ac signal.

concept of dropping the unwanted dynamic responses using the step structure design. This characteristic can be exploited to achieve a pure linear out-of-plane motion when the even modes of the actuator are suppressed. The actuator has undergone a reliability test by monitoring the variation of the dynamic characteristic after a continuous operation at its resonant frequency. It shows that the resonant frequency of the actuator has only $0.067 \%$ change after $10^{9}$ cycles of operation. Compared with the existing thermal actuators, the reliability improvement of the present actuator is mainly due to the single-crystal-siliconfilm material and the single-layer structure design.

In a similar manner, the odd modes of the actuator can be suppressed by adding only a single step at the midpoint of the beam. Thus, this single-step-bridge can be used for some applications which require pure torsional motion. The dynamic responses of the single-step-bridge structure are also fabricated and characterized, as shown in Fig. 13. Fig. 13(a) and (b) shows the measured dynamic responses that are driven by the PZT-shaker and the input ac signal, respectively. The results demonstrate that the odd modes were suppressed by the present single-step-bridge actuator. The measurement results also agree well with the simulation results that are shown in Table I. 


\section{ACKNOWLEDGMENT}

The authors would like to thank the Center for Nano Science and Technology, National Tsing Hua University, and the Nano Facility Center, National Chiao Tung University, for providing the fabrication facilities.

\section{REFERENCES}

[1] W. Riethmuller and W. Benecke, "Thermally excited silicon microactuators," IEEE Trans. Electron Devices, vol. 35, no. 6, pp. 758-763, Jun. 1988.

[2] J. H. Comtois and V. M. Bright, "Surface micromachined polysilicon thermal actuator arrays and applications," in Proc. Tech. Dig. Solid-State Sens. Actuators Workshop, Hilton Head Island, SC, Jun. 2-6, 1996, pp. 174-176.

[3] L. Que, J.-S. Park, and Y. B. Gianchandani, "Bent-beam electro-thermal actuators for high force applications," in Proc. MEMS, Orlando, FL, Jan. 1999, pp. 31-36.

[4] C. S. Pan and W. Hsu, "An electro-thermally and laterally driven polysilicon microactuator," J. Micromech. Microeng., vol. 7, no. 1, pp. 7-13, Mar. 1997.

[5] W. Wang, J. Fang, and K. Varahramyan, "Compact variable-focusing microlens with integrated thermal actuator and sensor," IEEE Photon. Technol. Lett., vol. 17, no. 12, pp. 2643-2645, Dec. 2005.

[6] M. Wu and W. Fang, "Integrated bi-directional focusing and tracking actuators in a monolithic device for a MEMS optical pick-up head," J. Micromech. Microeng., vol. 16, no. 7, pp. 1290-1297, Jul. 2006.

[7] T. Seki, M. Sakata, T. Nakajima, and M. Matsumoto, "Thermal buckling actuator for micro relays," in Proc. TRANSDUCERS, Chicago, IL, 1997, pp. 1153-1156.

[8] M. J. Sinclair, "A high frequency resonant scanner using thermal actuation," in Proc. MEMS, Las Vegas, NV, Jan. 2002, pp. 698-701.

[9] W.-C. Chen, C. Lee, C.-Y. Wu, and W. Fang, "A new latched $2 \times 2$ optical switch using bi-directional movable electrothermal H-beam actuators," Sens. Actuators A, Phys., vol. 123/124, pp. 563-569, 2005.

[10] J. H. Comtois and V. M. Bright, "Applications for surface-micromachined polysilicon thermal actuators and arrays," Sens. Actuators A, Phys., vol. 58, no. 1, pp. 19-25, Jan. 1997.

[11] T. Moulton and G. K. Ananthasuresh, "Micromechanical devices with embedded electro-thermal-compliant actuation," Sens. Actuators A, Phys., vol. 90, no. 1, pp. 38-48, May 2001.

[12] J. M. Maloney, D. S. Schreiber, and D. L. DeVoe, "Large-force electrothermal linear micromotors," J. Micromech. Microeng., vol. 14, no. 2, pp. 226-234, Feb. 2004.

[13] R. A. Conant and R. S. Muller, "Cyclic fatigue testing of surfacemicromachined thermal actuators," in Proc. ASME IMECE, Anaheim, CA, 1998, pp. 273-277.

[14] W.-C. Chen, C.-C. Chu, J. Hsieh, and W. Fang, "A reliable single-layer out-of-plane micromachined thermal actuator," Sens. Actuators A, Phys., vol. 103, no. 1, pp. 48-58, Jan. 2003.

[15] J. M. Gere, Mechanics of Materials, 5th ed. Pacific Grove, CA: Brooks/Cole, 2001.

[16] W. Fang and J. A. Wickert, "Post-buckling of micromachined beams," J. Micromech. Microeng., vol. 4, no. 3, pp. 116-122, Sep. 1994.

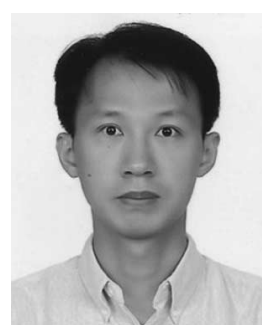

Wen-Chih Chen received the M.S. degree from National Tsing Hua University, Hsinchu, Taiwan, R.O.C., in 2001. He is currently working toward the $\mathrm{Ph} . \mathrm{D}$. degree in the Department of Power Mechanical Engineering, National Tsing Hua University.

He was an R\&D Engineer with Asia Pacific Microsystems Inc., developing optical MEMS components. His research interests include MEMS actuators and optical MEMS systems.

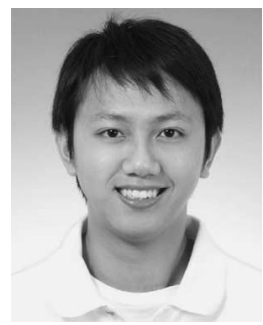

Po-I Yeh received the M.S. degree from the National Tsing Hua University, Hsinchu, Taiwan, R.O.C., in 2006.

He is currently with the Department of Power Mechanical Engineering, National Tsing Hua University. His research interests include MEMS thermal actuators and microfabrication.

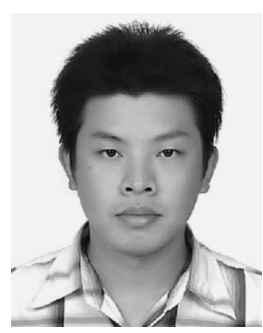

Chih-Fan Hu was born in Taipei, Taiwan, R.O.C., in 1982. He received the M.S. degree from the National Tsing Hua University, Hsinchu, Taiwan, R.O.C., in 2007.

$\mathrm{He}$ is currently with the Department of Power Mechanical Engineering, National Tsing Hua University. His research interests include MEMS actuators, pressure sensors, and flexible sensors.

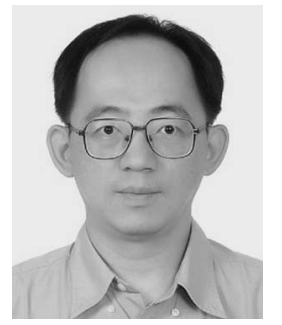

Weileun Fang (M'07) was born in Taipei, Taiwan, R.O.C., in 1962. He received the Ph.D. degree from Carnegie Mellon University, Pittsburgh, PA, in 1995. His Ph.D. research focused on determining the mechanical properties of thin films by using micromachined structures.

$\mathrm{He}$ was a Postdoctoral Researcher with the National Synchrotron Radiation Research Center, Hsinchu, Taiwan, in 1995. He joined the Department of Power Mechanical Engineering, National Tsing Hua University, Hsinchu, in 1996, where he is currently a Professor and a Faculty Member of the Institute of Nanoengineering and Microsystems. From June to September 1999, he was with the California Institute of Technology, Pasadena, as a Visiting Associate with Prof. Y.-C. Tai. He has established a MEMS testing and characterization laboratory. His research interests include MEMS, in particular microfabrication/packaging technologies, microoptical systems, microactuators, and the characterization of the mechanical properties of thin films.

Dr. Fang is a member of the American Society of Mechanical Engineers. 